\title{
Model Study of Cardiac Output Measurement by Thermodilution in Thermal Instability
}

\author{
M. Gawlikowski ${ }^{a, *}$, T. Pustelny ${ }^{a}$, B. Przywara-Chowaniec ${ }^{b}$ \\ AND J. NOWAK-GAWLIKOWSKA ${ }^{c}$ \\ ${ }^{a}$ Division of Mathematics and Physics, Silesian Technical University, Gliwice, Poland \\ ${ }^{b}$ Silesian Medical University, II Clinic of Cardiology, Zabrze, Poland \\ ${ }^{c}$ Specialized Hospital No. 1, Bytom, Poland
}

\begin{abstract}
Cardiac output is one of the most valuable hemodynamic parameter. It is measured commonly with intravascular pressures by pulmonary artery catheterization technique. The cardiac output itself is measured by dilution of thermal marker method (thermodilution), which is not resistant for various disturbances. In this paper the influence of blood temperature on metrological properties of thermal dilution method was described.
\end{abstract}

PACS numbers: 47.63.Cb, 87.19.U-

\section{Introduction}

Cardiac output (CO) is the most fundamental hemodynamic parameter, which defines function of heart muscle. $\mathrm{CO}$ value may be subject to change both in physiological and pathological condition of patient $[1,2]$. Accurate measurement of $\mathrm{CO}$ allows to illness diagnose and determinants of its treatment.

There are two principal methods of $\mathrm{CO}$ measurement: the Fick method and thermodilution [3, 4]. Because of complicated examination procedure first of themes is most often applied in research activity. During the last 30 years thermodilution has became the "gold clinical standard" in hemodynamic monitoring [3, 4]. Measurement of cardiac output and other hemodynamic parameters (mainly intravascular pressures) is realized by pulmonary artery catheterization (PAC) and the Swan-Ganz catheter usage $[3,5]$. It is inserted from jugular vein, through the right atrium and right ventricle to pulmonary trunk. Miniaturized temperature-sensitive resistor is located at the catheter tip (Fig. 1). Isotonic salt solution (iced or room temperature) is injected via catheter's duct to right atrium, which causes local blood temperature drop in cardiac chambers and distant pulmonary vessels. The local blood temperature in pulmonary artery is registered and measured by computer system. This temperature vs. time dependence is called indicator dilution curve (IDC). From IDC, by the Stewart-Hamilton formula [2] CO is calculated

* corresponding author; e-mail:

maciej.gawlikowski@poczta.onet.pl

$$
\mathrm{CO}=\frac{c_{\mathrm{i}} \rho_{\mathrm{i}}}{c_{\mathrm{b}} \rho_{\mathrm{b}}} \frac{V_{\mathrm{i}}\left(T_{\mathrm{b}}-T_{\mathrm{i}}\right)}{\int_{t_{0}}^{\infty}-T(t) \mathrm{d} t}
$$

$c_{\mathrm{i}}, c_{\mathrm{b}}$ - specific heat of indicator and blood, $\rho_{\mathrm{i}}, \rho_{\mathrm{b}}$ - specific mass of indicator and blood, $V_{\mathrm{i}}$ - volume of indicator, $T_{\mathrm{b}}, T_{\mathrm{i}}$ - temperature of blood and indicator, $T(t)$ - IDC.

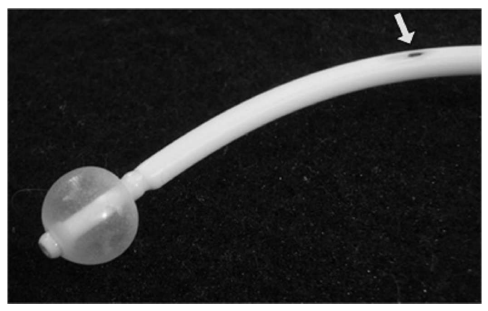

Fig. 1. Tip of Swan-Ganz catheter (thermal-sensitive resistor was marked by arrow).

Thermal-sensitive resistor (made of platinum or - in contemporary construction - of nickel) is pre-calibrated for operating blood temperature at $37.0^{\circ} \mathrm{C}[6-8]$. In many cases (e.g. after surgery or during intra-operation hypothermia) blood temperature may be instable, which may affect the accuracy of IDC measurement and CO estimation.

\section{Goal}

Cardiac output measurement by thermodilution is recognized as sensitive to numerous physical and biological disturbances, therefore the accuracy of this method is limited to about $20 \%[5,9,10]$. 
The goal of work was cardiac output vs. blood temperature dependence assessment as a potential method's accuracy limitation factor.

\section{Material and methods}

Investigations were performed on physical model of pulmonary circulation (Fig. 2). This model is able to simulate the principal features of cardiovascular system, which are significant for thermodilution $\mathrm{CO}$ examination. Among the physical phenomena it allows us to simulate blood viscosity, mass density and specific heat. It is possible to modeling the selected anatomical features of pulmonary circulation: atrium and ventricle volumes, pulmonary arteries diameters and valves operation (including malfunctions) $[6,11]$. Model is able to generate pulsating flow, rhythmical or irregular.

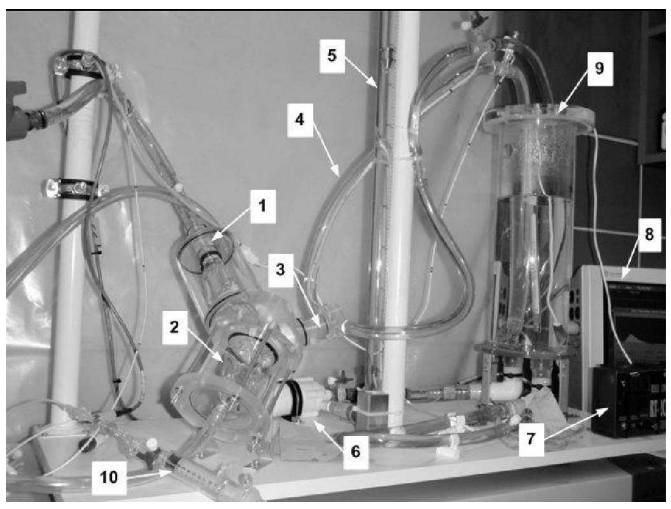

Fig. 2. Physical model of pulmonary circulation. $1-$ atrium, 2 - ventricle, 3 - pulmonary trunk, 4 - pulmonary artery, 5 - rotameter, 6 - centrifugal pump, 7 - temperature regulator, 8 - patient monitor, 9 pulsating-to-continouous flow converter, 10 - thermal indicator application syringe.

Pulsating flow generated by heart model was converted to continuous flow in hydraulic system, consisted of tank (Fig. 2, 9) and centrifugal pump (Fig. 2,6). The standard cone-and-float meter (accuracy class 1.5) was utilized to average volumetric flow assessment.

Cardiac output was measured by "cold" and "room temperature" thermodilution method. Indicator temperature was measured at the outlet of syringe. During experiments the following equipment was utilized:

- B2269 Swan-Ganz catheter (Burron Medical),

- PM-9000 (Mindray) patient monitor with CO measurement module.

Thermal instability of patient was defined as a middle hypothermia with blood temperature in the range of $25-30{ }^{\circ} \mathrm{C}$ [11]. Investigations were performed by comparison method: measured vs. reference flow dependences in state of thermal instability were compared with corresponding dependences obtained in normal blood temperature state.

\section{Results}

For middle hypothermia the liquid temperature measured at the same point of model by various apparatuses were:

- $27.07^{\circ} \mathrm{C}$ (reference thermometer Fluke 1523 equipped with PRT probe),

- $25.90^{\circ} \mathrm{C}$ (Swan-Ganz catheter and PM-9000 patient monitor).

Relative temperature measurement unreliability was $-4.2 \%$ (in comparison with reference apparatus). For control group liquid temperature was $37.0^{\circ} \mathrm{C}$.

For "room temperature" thermodilution $\left(T_{\mathrm{i}}=\right.$ $21.5-22.1^{\circ} \mathrm{C}$ ) and average flows in the range of $1.7-3.2 \mathrm{~L} / \mathrm{min}$ it was not possible to measure CO by patient monitor (error was reported). For reference flow $4.0 \mathrm{~L} / \mathrm{min}$ the result obtained by thermodilution method was $5.76 \pm 0.25 \mathrm{~L} / \mathrm{min}$.

For "iced" thermodilution $\left(T_{\mathrm{i}}=0.5-3.5^{\circ} \mathrm{C}\right)$ the measured vs. reference flow dependences were depicted in Fig. 3.

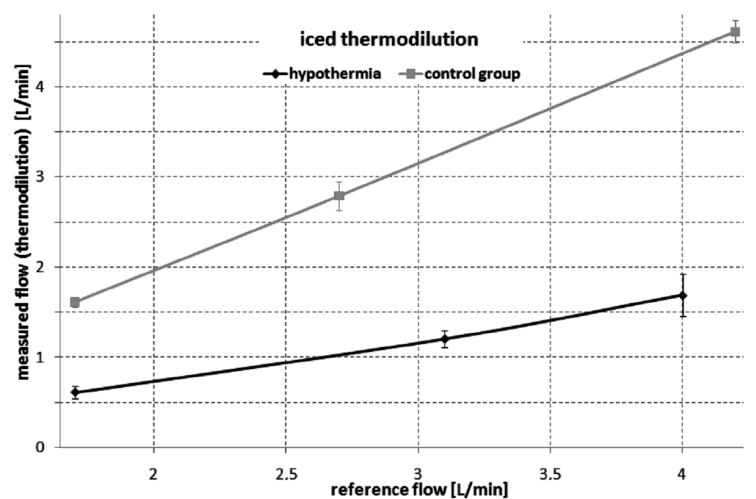

Fig. 3. Cardiac output measurement by iced thermodilution - the comparison of hypothermia and control group.

Linear relationship between reference and measured data justified performing analysis by linear regression method (Table).

TABLE

Linear regression coefficients comparison of hypothermia and control group.

\begin{tabular}{l|c}
\hline \hline & Regression coefficient \\
\hline hypothermia $\left(T=27.0^{\circ} \mathrm{C}\right)$ & 0.46 \\
control group $\left(T=37.0^{\circ} \mathrm{C}\right)$ & 1.20
\end{tabular}




\section{Discussion}

Because of significant deviations of flow measurements for hypothermia the system inspection was performed and the examinations were repeated. In both experiments obtained results were similar. By this way measurement system and examination technique were positively validated.

The explanation of observed effect may be overestimation of integral value in denominator of dilution equation (Eq. (1)). Thermal sensitive element in the Swan-Ganz catheter is calibrated for standard blood temperature [8] and its polynomial transfer function is approximated by linear function at neighbourhood of a point $37^{\circ} \mathrm{C}$. Therefore significant decreasing of base blood temperature causes change of thermoelement operating point and - in consequence - linearization of transfer function may introduce additional unreliability. To prove this assumption precise measurement of thermoelement transfer function was performed (Fig. 4).

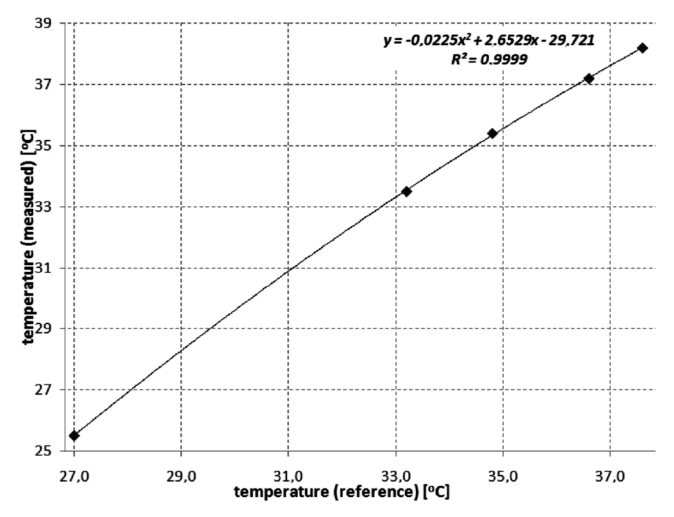

Fig. 4. Thermoelement transfer function.

Slope of linear approximation function is given by following equation:

$$
\frac{\mathrm{d} T_{\text {measured }}}{\mathrm{d} T_{\text {reference }}}=-0.045 T_{\text {reference }}+2.6529
$$

and equals (for $37^{\circ} \mathrm{C}$ and $27^{\circ} \mathrm{C}$, respectively):

$$
\begin{aligned}
& \left.\frac{\mathrm{d} T_{\text {measured }}}{\mathrm{d} T_{\text {reference }}}\right|_{T=37^{\circ} \mathrm{C}}=0.98 . \\
& \left.\frac{\mathrm{d} T_{\text {measured }}}{\mathrm{d} T_{\text {reference }}}\right|_{T=27^{\circ} \mathrm{C}}=1.43 .
\end{aligned}
$$

Significantly higher (by 46\%) slope of linear approximation function is cause of integral overestimation in the Stewart-Hamilton formula (Eq. (1)) and underestimation of CO measurement by thermodilution.

In practice for every catheter the calibration factor is given (usually in operation manual) $[5,7,8]$. It must be entered to the patient monitor before beginning of $\mathrm{CO}$ measurement procedure. This factor contains components dependent on heat exchange between thermoelement and environment, indicator temperature and technology of manufacturing. For investigated catheter calibration factor equals 0.802 and 0.872 (for "iced" and "room temperature" thermodilution, respectively). Mentioned factor is estimated for normal blood temperature and its value for blood thermal instability is unknown.

\section{Conclusions}

Performed model investigations pointed out on significant sensitivity of $\mathrm{CO}$ measurement by thermodilution method to variations of base blood temperature.

Presented conclusion has important clinical meaning and - in Authors' view - should be taken into consideration during hemodynamic monitoring of patients in thermal instability.

\section{Acknowledgments}

This work was supported by Polish Ministry of Science (grant No. N N518 336135) and Czesław M. Rodkiewicz Scholarship Foundation.

\section{References}

[1] A. Szczeklik, The internal diseases. Vol. I, The diseases of cardiovascular system, Practical Medicine Publishing, Kraków 2005 (in Polish).

[2] K.L. Zierler, Circul. Res. 10, 393 (1962).

[3] D. Payen, E. Gayat, Critical Care Suppl. 3, S-7 (2006).

[4] N.S. Nieminen, Polish Cardiology 63, 2 (2005) (in Polish).

[5] J.M. Headley, Invasive Hemodynamic Monitoring: Physiological Principles and Clinical Applications, Edwards Lifescience, Irvine (CA) 2002.

[6] A. Bochenek, The Anatomy of Human, Vol. II, PZWL, Warsaw 1992 (in Polish).

[7] T. Nishikawa, S. Doshi, Canad. J. Anesthesia 40, 142 (1993).

[8] G. Konieczny, Z. Opilski, T. Pustelny, E. Maciak, Acta Phys. Pol. A 116, 344 (2009).

[9] J.G. Webster, Encyclopedia of Medical Devices and Instrumentation, Wiley-Interscience, Hoboken 2006.

[10] B. Przywara-Chowaniec, L. Polonski, M. Gawlikowski, T. Pustelny, Acta Phys. Pol. A 116, 380 (2009).

[11] M. Gawlikowski, T. Pustelny, B. Przywara-Chowaniec, P. Struk, Acta Phys. Pol. A 114, A-79 (2008). 